\title{
Black Holes in Active Galactic Nuclei
}

\author{
Greg Madejski \\ Stanford Linear Accelerator Center, 2575 Sand Hill Rd, Menlo Park, CA USA
}

\begin{abstract}
The most viable scenario for modelling of active galactic nuclei includes a supermassive black hole (with the mass of $10^{6}-10^{9}$ Solar masses) accreting the galaxian matter from its vicinity. The release of the gravitational energy of the infalling matter is ultimately responsible for the large radiative output of those objects, and in some cases, for formation and acceleration of powerful relativistic jets. This paper outlines our current understanding of the structure of AGN, and summarizes the observational evidence for black holes in those objects.
\end{abstract}

\section{Introduction}

What are Active Galactic Nuclei? These celestial objects, originally found in the early sixties as point-like radio emitters, were identified with apparently stellar sources, possessing somewhat unusual spectra, with prominent emission lines. The identification of these line series as redshifted systems by Schmidt (1963) implied that quasars are distant and extremely luminous, commonly producing radiation at a rate of $10^{46} \mathrm{erg} \mathrm{s}^{-1}$; this is a hundred times or more in excess of the total luminosity of all the stars in a galaxy. Subsequent sensitive imaging of the nebulosities which often surround them implied that quasars are nuclei of galaxies, and thus are higher-luminosity counterparts of the compact nuclei of the so-called Seyfert galaxies (studied some twenty years before the discovery of quasars) as unusual emission line objects. We currently believe that they represent respectively the lower and higher luminosity end of the same population. Sensitive optical searches for point-like, blue objects revealed that quasars are quite numerous. However, only about a tenth of quasars found via optical searches might have bright point-like radio source associated with them. Importantly, in many (if not in most) cases, flux detected from quasars is time-variable in all observable bands, on time scales from years to days. This of course implies that the tremendous luminosity produced by a quasar originates in a relatively compact volume. The flux variability is random (non-periodic), and there are no confirmed reports of any periodicity in AGN.

A variety of scenarios were advanced to explain their nature, but the proposal that quasars are powered by an accretion of surrounding matter onto a black hole, advanced shortly after their discovery by Salpeter (1964) and Zeldovich \& Novikov (1964) became the paradigm that we are developing and testing today. While this is a viable and very attractive paradigm, only the last few years brought a solid evidence for it, allowing also to measure the mass of the central

Work supported in part by the Department of Energy contract DE-AC03-76SF00515.

Invited talk presented at the Dark Matter in Astro- and Particle Physics (DARK-2002), 
object. It is important to note here that quasars are much more numerous at a redshift $z \sim 2$ than they are locally, meaning that a substantial fraction of galaxies must have undergone the quasar phase. With this, it is likely that many otherwise normal galaxies harbor supermassive black holes, "dead quasars," and such objects are certainly providing at least equal if not greater contribution towards the total census of dark matter as do truly active galaxies. For a more detailed discussion on this subject, the reader is referred to the contribution of D. Merritt in these proceedings. It is worth noting here that we currently have a good evidence for a presence of numerous, much less massive black holes (with masses of a few Solar masses) in our Galaxy and also in other galaxies. Those are members of binary stellar systems, and are likely to be end products of stellar evolution; for a recent review of this subject, see, e.g., Charles (1998).

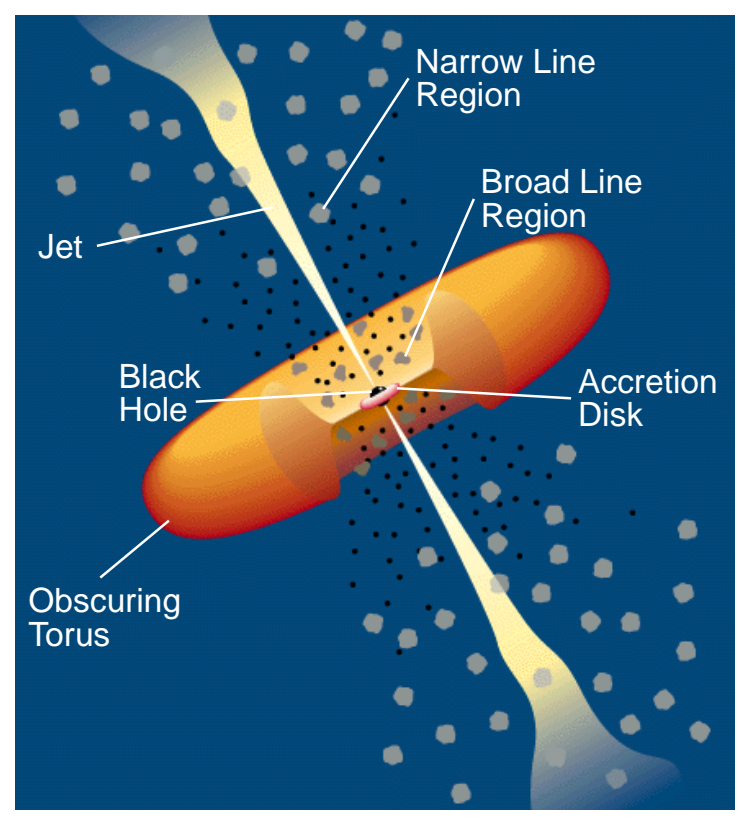

Fig. 1. Schematic picture of an active galaxy, including its ingredients: the accreting black hole, accretion disk, and obscuring torus. In some cases, an active galaxy possesses a relativistic jet, which, when pointing close to the line of sight, is Doppler-boosted and thus dominates the observed radiation. If the jet is absent, the isotropic components (such as the emission line light, and flux from the accretion disk) dominate the observed flux (figure courtesy of European Space Agency)

We currently believe that most likely we observe two rather distinct aspects electromagnetic emission in various active galaxies, one due to a relativistic jet, and another due to an isotropic component, and depending on an object, one or the other may dominate the observed flux. Detailed radio-interferometric 
studies, allowing angular resolution of $10^{-3}$ arc seconds, reveal jet-like structures, which in some cases, are variable in time, implying so-called "super-luminal expansion" of the inner regions (for a recent review, see Jorstad et al. 2001). This nomenclature arose because the apparent rate of angular separation of individual components of the jet times the distance to the object exceeded the speed of light. The most viable explanation of such a behavior invokes relativistic motion of the emitting matter at an angle close to the line of sight, where the "superluminal" motion is just a projection effect. Such a scenario implies that the accretion onto a black hole is in some cases associated with launching of a jet of material that is ultimately producing the observed radiation.

An important consequence of the relativistic motion of the jet close to the line of sight is strong Doppler boost of the emitted radiation, which, with the bulk Lorentz factor of the jet $\Gamma \sim 10$, could result in the flux a thousand times brighter than it would appear in its rest frame. The positional coincidence of many quasars detected by the EGRET instrument flown on the Compton Gammaray Observatory satellite (sensitive roughly in the $100 \mathrm{MeV}-10 \mathrm{GeV}$ range) with jet-dominated radio-emitting quasars led many workers to conclude that the entire electromagnetic emission in those objects (often known as "blazars") arises in the Doppler-boosted jet aligned closely to the line of sight. Several such blazars are routinely detected up to the $\mathrm{TeV}$ gamma-ray range via ground-based Cerenkov telescopes. Our current best scenario for the radiative processes operating in the jet has the radio-band through the soft X-ray emission arising via the synchrotron process. Within this model, the gamma-ray flux is produced via Compton-upscattering of lower energy photons by the same electrons that are responsible for the synchrotron emission; the seed photons might be internal to the jet, or due to the circum-nuclear environment of the host galaxy. Since a particular orientation is needed for a quasar to be labelled as a blazar, there must be many more "mis-directed" blazars. In fact, we currently believe that if the jet points farther away from the line of sight, an object is most likely a radio galaxy (a strong, diffuse radio emitter often showing large-scale jets in the plane of the sky), with a compact but rather faint core.

Not all quasars possess luminous jets. Many quasars discovered via optical searches are devoid of compact radio emission, and in fact show a variety of features such as variable yet relatively symmetric optical and UV emission lines, indicating an absence of Doppler boost and thus implying nearly-isotropic emission. While the jet-dominated sources are interesting in their own right, it is the study of isotropic radiation that appears to lead to more conclusive inferences about the nuclei themselves, and provides the evidence for existence of black holes in active galaxies. Our understanding of the fundamental processes operating in AGN is far from complete, but we are reasonably confident that in all cases, the ultimate source of power is accretion - via some form of an accretion disk - onto black holes with masses ranging from $\sim 10^{6}$ to $\sim 10^{9} M_{\mathrm{o}}$, where $M_{\mathrm{o}}$ is the mass in the units of Solar mass. This follows rather readily from Eddington luminosity arguments: an object radiating at the total luminosity $L$ must have a mass $M$ such that $M>L \sigma_{T} / 4 \pi G m_{p} c$ (where $\sigma_{T}$ is the Thomson cross-section 
and $m_{p}$ is the mass of proton), or, $M / M_{\mathrm{o}}>L /\left(1.3 \times 10^{38} \mathrm{erg} \mathrm{s}^{-1}\right)$. This means that a quasar with $L \sim 10^{44} \mathrm{erg} \mathrm{s}^{-1}$ has to have the central object with mass of at least $\sim 10^{6} M_{\mathrm{o}}$.

\section{Observational Evidence for Black Holes in AGN}

As mentioned above, AGN are rapidly variable in all accessible energy bands (see, e.g., Krolik, Done, \& Madejski 1993). The causality arguments imply that the most rapid flux variability arises from smallest physical regions, and since generally the shortest time scales are seen in the $\mathrm{X}$-ray band, this is probably our best probe of the nucleus. Nonetheless, the evidence for the existence of black holes in AGN comes from all observable bands; this relies mainly on kinematics of material moving in the resultant strong gravitational field, or even gravitational redshift.

\subsection{Optical and IR data}

The precise spatially-resolved Hubble Space Telescope spectroscopy of nearby AGN indicates that the gas in the very close (tens of parsecs) vicinity of nuclei moves at high velocities in opposite directions on opposite sides of the nucleus. Well-known is that of M 87 (Harms et al. 1994; cf. Fig. 2), but this also has been seen in many galaxies with no pronounced activity (see a review by Ford et al. 1998). Ford et al. (1998) and Ho et al. (1998) (see also the contribution by D. Merritt in these proceedings) suggest that as many as a half of all galaxies contain supermassive black holes. Even our own Galaxy is likely to also harbor a relatively inactive one, with a mass of $\sim 3 \times 10^{6} M_{\mathrm{o}}$ (cf. Eckart \& Genzel 1997; see the contribution by F. Baganoff in these proceedings). Interestingly, M 87 shows a relativistic jet, visible in the radio, optical, and X-ray bands. While it is not a true blazar, it's likely to be a case of a jet pointing sufficiently far away from our line of sight that it's probably an example of a "misdirected" blazar, and thus it may provide the best direct evidence for an association of black holes with blazars.

\section{$2.2 \quad$ VLBI radio data}

High angular resolution, Very Long Baseline Interferometry (VLBI) radio studies provided perhaps the most elegant evidence for supermassive black holes in AGN. These came from mapping of $\mathrm{H}_{2} \mathrm{O}$ megamaser spots, most commonly observed in Seyfert 2 - type active galaxies, which are believed to be quasars seen in (or close to) the plane of the accretion disk. Perhaps the best publicized case is of the low-luminosity object NGC 4258. The data (Miyoshi et al. 1995; see Fig. 3) imply that the spot-emitting material is confined to a flattened, disklike structure, and the spatial velocity distribution is nearly perfectly Keplerian. Any other distribution of mass such as massive dark objects would disperse on a relatively short time scale (cf. Maoz 1995), leaving us with a black hole with a 


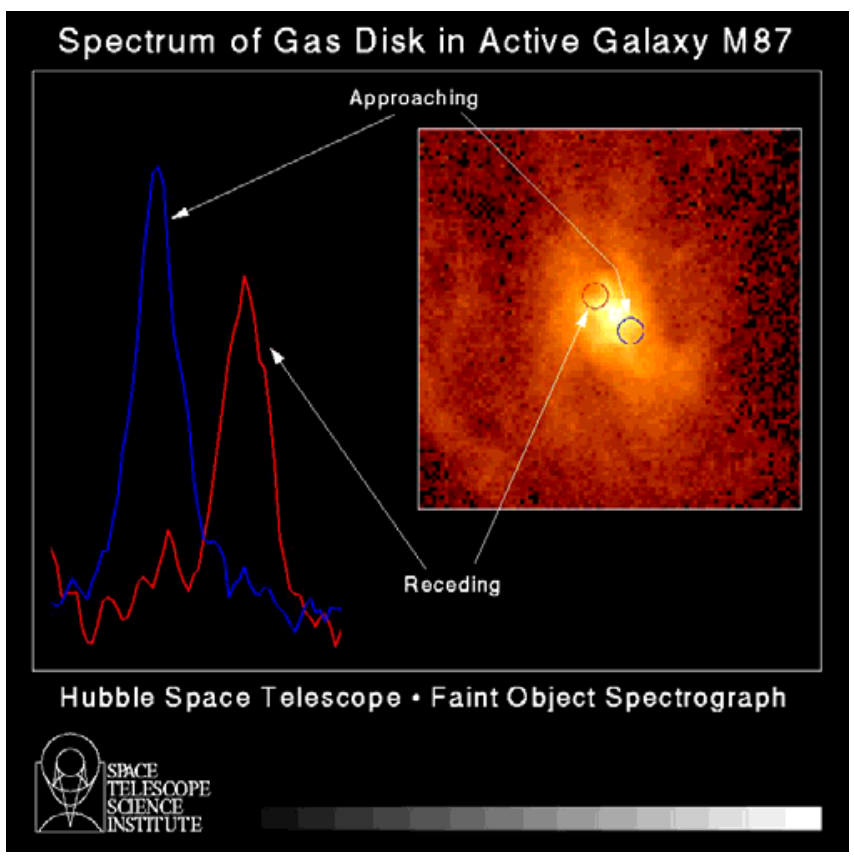

Fig. 2. Hubble Space Telescope image of the radio galaxy M 87, showing the velocity profile of emitting gas near the nucleus (STScI Public Archive). The data imply a mass of the central object of $\sim 3 \times 10^{9} M_{\text {o }}$

mass of $\sim 3.6 \times 10^{7} M_{\mathrm{o}}$. Given the limited sensitivity of VLBI observations, there are currently only a few megamaser AGN with measured black hole masses; one example is NGC 4945, discussed in more detail below (see Fig. 4).

\section{$2.3 \quad \mathrm{X}$-ray observations}

As mentioned above, in the active galaxies believed to emit their radiation nearly isotropically, the largest amplitude and most rapid variability is observed in the $\mathrm{X}$-ray band. The best early data were collected with the European EXOSAT satellite, and those observations clearly indicated variability on time scales as short as $10,000 \mathrm{~s}$ from objects with X-ray luminosity on the order of $10^{43} \mathrm{erg}$ $\mathrm{s}^{-1}$. Assuming isotropy of the X-ray emission, it's hard to envision any other form of mass condensation besides an accreting black hole that could produce as much light from such a small volume. Current observations with various $\mathrm{X}$-ray 


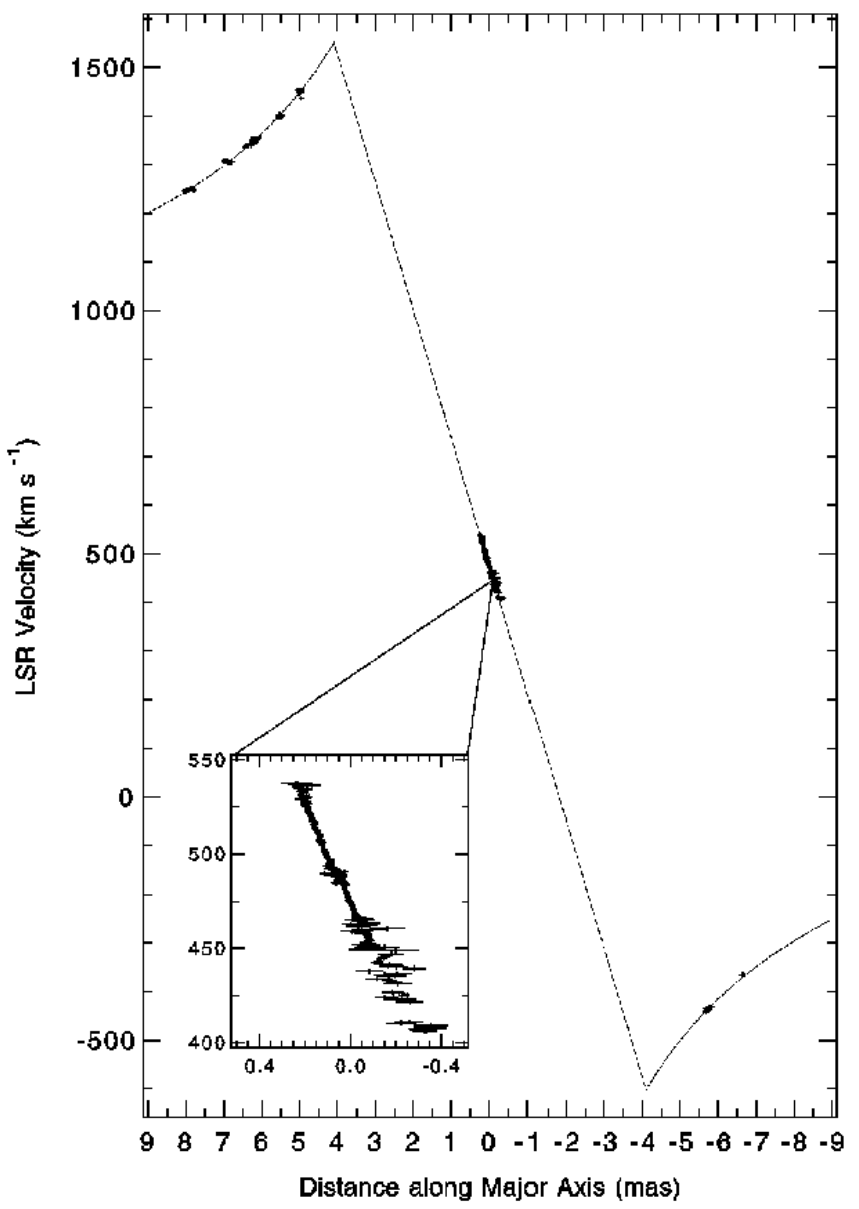

Fig. 3. The distribution of megamaser spots in the Seyfert 2 galaxy NGC 4258, which is seen close to the plane of the accretion disk (from Miyoshi et al. 1995). The run of velocity with distance from the central source is very close to Keplerian, implying that the most of the mass of $3.6 \times 10^{7}$ Solar masses is enclosed well within the radius of $\sim 0.13$ parsec

satellites (see, e.g., Markowitz \& Edelson 2001) confirm this picture: large amplitude X-ray variability on time scales as short as a few hours are commonplace amongst AGN.

The best candidate for the ultimate energy source in active galaxies is accretion onto a black hole, but what physical processes might be responsible for the observed overall electromagnetic emission? An excellent monograph on the subject is "Active Galactic Nuclei" by J. Krolik. In summary, the accreting matter can release its gravitational energy relatively readily - through radiation but the angular momentum of the accreting matter must also be dissipated or 


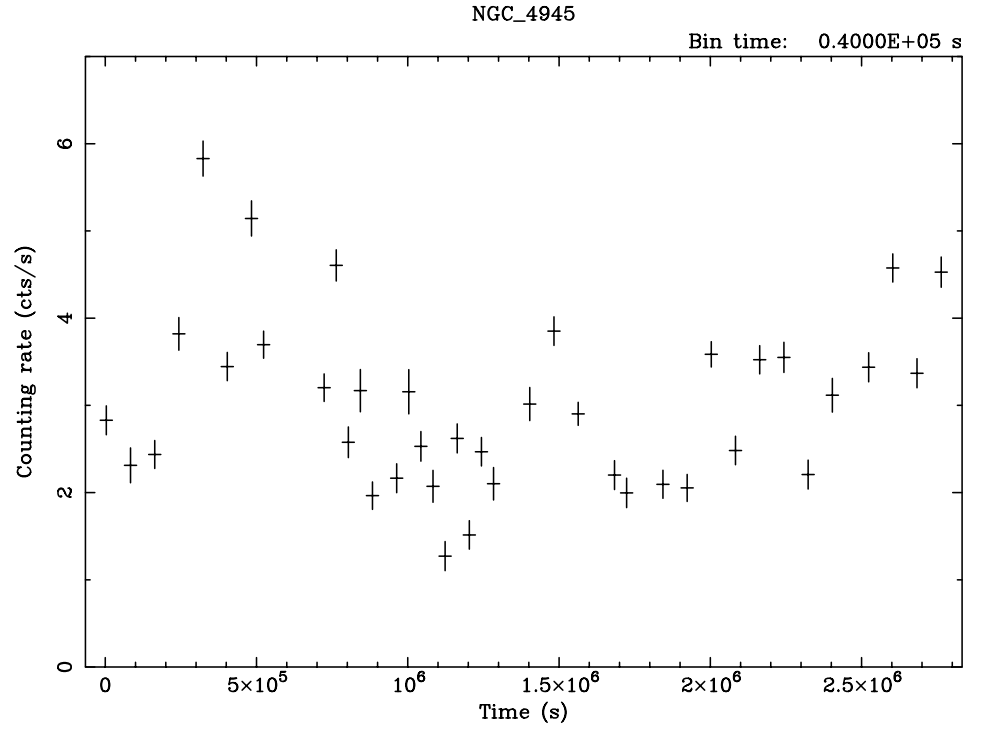

Fig. 4. History of the X-ray flux from the active galaxy NGC 4945 as measured with the Rossi X-ray Timing Explorer satellite in 1997 (from Madejski et al. 2000). This object is a megamaser source, with the measured mass of the central object of $\sim 1.4 \times 10^{6}$ Solar masses (Greenhill, Moran, \& Herrnstein 1997), which, when interpreted as a black hole, would have a Schwarzchild radius $R_{S}=2 G M / c^{2}$ of $\sim 4 \times 10^{11} \mathrm{~cm}$. The X-ray flux time series reveals large amplitude variability on time scales of $10 \mathrm{ksec}$, indicating that the emission most likely originates well within a few hundred Schwarzchild radii

carried away. This naturally leads to a flattened structure, the accretion disk. Many detailed calculations of the accretion process (originating with the seminal paper by Shakura \& Sunyaev 1973) imply that the release of gravitational energy leads to heating of the accretion disk; a good source covering this subject is "Accretion Power in Astrophysics" by Frank, King, and Raine. Since the density of matter in the disk is calculated to be relatively large (in excess of $10^{10}$ particles $\mathrm{cm}^{-3}$ ), Coulomb interactions are a relatively efficient mechanism producing radiation, and the material in the disk emits a spectrum which is quasi-thermal. However, the maximum temperature of such an accretion disk for a black hole with a mass of $10^{6}$ Solar masses is less than $1 \mathrm{keV}$. Since many active galaxies are very strong $\mathrm{X}$-ray emitters up to $50-100 \mathrm{keV}$, another process must be invoked to account for the production of $\mathrm{X}$-rays. The details of this are poorly known, but the best current scenario has the dissipation of the gravitational energy via viscous processes which heat the disk, but which are also associated with formation of strong magnetic fields. This scenario is somewhat analogous to the Solar corona: such fields undergo reconnection, where electrons might be accelerated to trans-relativistic energies. Since the thermal emission from the disk provides ample soft photons, these photons can be easily Compton-upscattered by the 
corona, and are observed as the hard X-ray emission (for a recent summary, see Zdziarski et al. 1997).

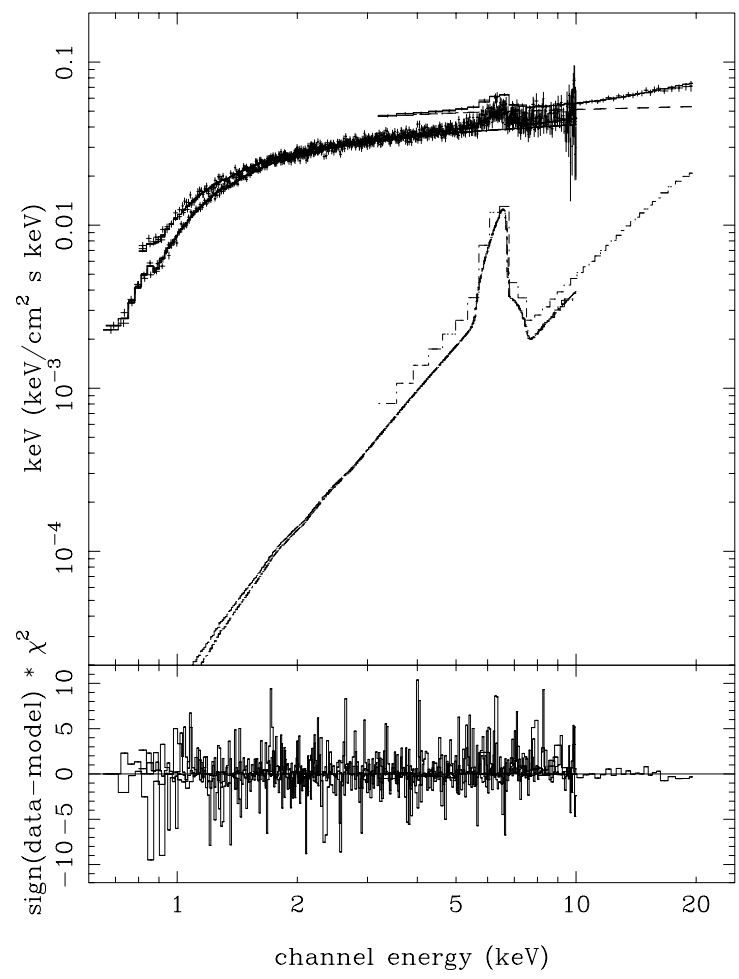

Fig. 5. The X-ray spectrum of a bright active galaxy IC 4329a, which is a good representative of the Seyfert class. The spectrum is absorbed at low energies by photoelectric absorption due to the interstellar medium of the host galaxy, but above $\sim 1 \mathrm{keV}$ it shows strong intrinsic $\mathrm{X}$-ray emission, well-described as a power law with a photon number index of $\sim 2$. The primary power law, which at high energies (at $\sim 200 \mathrm{keV}$, beyond that shown in the Figure) rolls off steeply, is presumably produced by thermal Comptonization of UV photons produced in the accretion disk by electrons with $k T \sim 200 \mathrm{keV}$ and optical depth of nearly unity. The discrete spectral feature at $\sim 6.4$ $\mathrm{keV}$ is the fluorescence line of neutral iron, with the profile somewhat distorted by gravitational and kinematic effects. The excess flux over the power law above $10 \mathrm{keV}$ is best interpreted as due to Compton reflection of the primary power law by the material in the cold accretion disk (from Done, Madejski, \& Zycki 2000)

Importantly, the cold accretion flow reprocesses the primary X-ray continuum which illuminates it: the hard photons are Compton-reflected from the cold gas, modifying the continuum (cf. Lightman \& White 1988; see Fig. 5). Since iron is a relatively abundant element in the Cosmos, yet has a relatively high fluorescence yield, the strongest discrete spectral feature predicted and observed 
from accretion disks is the iron $\mathrm{K} \alpha$ fluorescence line at $6.4 \mathrm{keV}$. Its profile should reflect the kinematics of the flow, and indeed, probably the most compelling evidence for cold matter moving in a very strong gravitational field of an AGN came from the ASCA data for the Seyfert galaxy MCG-6-30-15 (Tanaka et al. 1995; cf. Fig 6). The profile of the line shows two prongs, from the receding and approaching material in the accretion disk, but more importantly, a pronounced "red" wing, best explained by the line photons affected by strong gravity, including redshift (cf. Fabian et al. 1989). This implies emission at $<10$ Schwartzchild radii $r_{\mathrm{S}}$, close to the last stable orbit around a black hole. ASCA data (cf. Nandra et al. 1987) reveals a few other AGN showing Fe K lines with similar profiles; good examples are NGC 3516 and NGC 3783. The importance of detailed measurements of those lines lies of course in the fact that their profiles potentially probe the regions closest to the black hole, a few to few tens of $R_{\mathrm{S}}$, while the megamaser and circumnuclear gas studies discussed above reach to a fraction of a parsec, on the order of a thousand $R_{\mathrm{S}}$.

\section{Summary}

In summary, the recent astrophysical observations in radio, optical and $\mathrm{X}$-ray bands provide us with quite good evidence that accretion of matter onto supermassive black holes is the best scenario explaining the source of energy in active galaxies. The current data allow us to measure the masses of black holes in a number of active galaxies, and estimate them in many more. A number of current or planned satellite-based observatories - such as Chandra, XMM-Newton, Constellation-X, and GLAST - are bound to provide new data to allow the study of strong gravity provided by those objects.

\section{References}

1. Charles, P. 1998, in Theory of Black Hole Accretion Disks, eds. M. Abramowicz, G. Bjornsson, \& J. Pringle, Cambridge University Press, 1

2. Done, C., Madejski, G., \& Zycki, P. 2000, ApJ, 536, 213

3. Eckart, A., \& Genzel, R. 1997, MNRAS, 284, 576

4. Fabian, A., Rees, M., Stella, L., \& White, N. 1989,MNRAS, 238, 729

5. Ford, H., Tsvetanov, Z., Ferrarese, L., \& Jaffe, W. 1998, in The Central Regions of the Galaxy \& Galaxies, IAU Symp. 184

6. Frank, J., King, A., \& Raine, D. 1992, Accretion Power in Astrophysics, Cambridge University Press

7. Greenhill, L., Moran, J., \& Herrnstein, J. 1997, ApJ, 481, L23

8. Harms, R., et al. 1994, ApJ, 435, L35

9. Ho, L., 1998, in The Central Regions of the Galaxy and Galaxies, IAU Symp. 184

10. Jorstad, S., Marscher, A. P., Mattox, J., Wehrle, A., Bloom, S., \& Yurchenko, A. 2001, ApJS, 134, 181

11. Krolik, J. 1999, Active Galactic Nuclei, Princeton University Press

12. Krolik, J., Done, C., \& Madejski, G. M. 1993, ApJ, 402, 432

13. Lightman, A., \& White, T. 1988, ApJ, 335, 57 


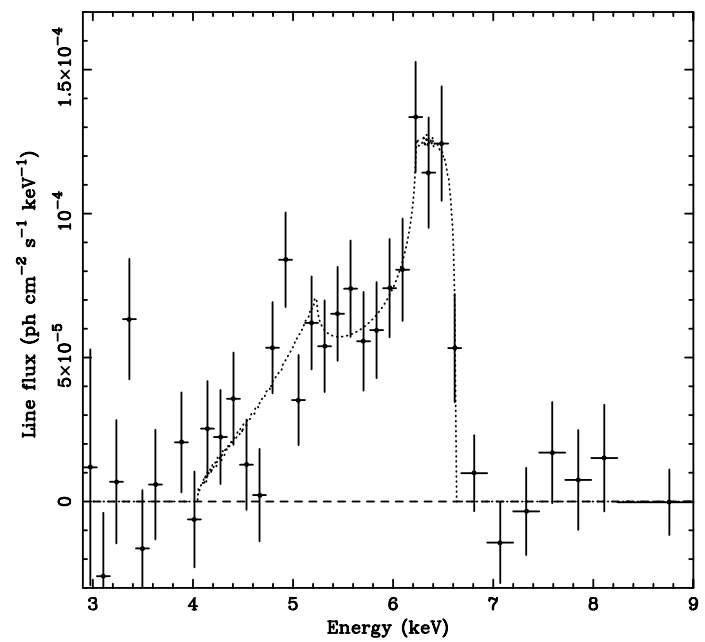

Fig. 6. The profile of the Fe K line, presumably originating due to fluorescence of iron present in the neutral accreting material irradiated by the primary $\mathrm{X}$-ray power law. The distorted profile of the line is best explained as due to gravitational and kinematic effects on the material revolving in an accretion disk located in a strong gravitational field such as due to a black hole (from Tanaka et al. 1995)

14. Madejski, G., Zycki, P. Done, C., Valinia, A., Blanco, P., Rothschild, R., \& Turek, B. 2000, ApJ, 535, L87

15. Maoz, E. 1995, ApJ, 447, L91

16. Markowitz, A., \& Edelson, R. 2001, ApJ, 547, 684

17. Miyoshi, M., et al. 1995, Nature, 373, 127

18. Nandra, K., George, I., Mushotzky, R., Turner, T. J., \& Yaqoob, T. 1997, ApJ, 477, 602

19. Salpeter, E. E. 1964, ApJ, 140, 796

20. Schmidt, M. 1963, Nature, 197, 1040

21. Shakura, N., \& Sunyaev, R. 1973, A\&A, 24, 337

22. Tanaka, Y., et al. 1995, Nature, 375, 659

23. Zdziarski, A., et al. 1997, in The Transparent Universe, eds. C. Winkler et al. (ESA: Paris), SP-382, 373

24. Zeldovich, Y., \& Novikov, I. 1964, Sov. Phys. Dokl., 158, 811 